\title{
Synthesizing Robust Mode Shapes with $\mu$ and Implicit Model Following
}

\author{
John C. Morris \\ Electrical Engineering Dept. \\ Caltech, Mail Code 116-81 \\ Pasadena, CA 91125 (USA)
}

\author{
Pierre Apkarian \\ CERT DERA \\ Control Engineering Dept. \\ 2 avenue Edouard Belin (BP 4025) \\ 31055-Toulouse Cedex (France)
}

\section{Abstract}

New control synthesis problems involving assignment of closed-loop modal shapes using implicit model following (IMF) structure are considered in the context of $\mathcal{H}_{2}, \mathcal{H}_{\infty}$, and $\mu$-synthesis theories. An extension to the dynamic output feedback case is first given for the well-known quadratic or $\mathcal{H}_{2}$ IMF problem. The IMF problem is then embedded within the framework of $\mu$ control theory and extensions for including uncertainty discussed. A robust synthesis methodology is presented using $\mu$ theory. Finally, an application of the robust IMF synthesis methodology to modal shape assignment for the longitudinal axis of a helicopter is demonstrated.

\section{Introduction}

The purpose of this paper is to study the problem of achieving precise and robust time domain specifications on specific states of a closed-loop system using frquency-domain robust control design methods, and in particular $\mu$-synthesis. While most standard methods for robust control design focus on frequency domain specifications, many performance specifications in aerospace applications are explic itly stated in the time domain in terms of settling times, damping ratios, and internal state decoupling. Thus the application of robust control design methods can lead to a large amount of "trial and error" before obtaining satisfactory conventional specifications in terms of time domain properties. While it may be worthwhile to encourage design engineers to reconsider conventional specifications, it would be immediately useful if frequency domain robust control methods could be modified to handle conventional specifications. The latter is the approach taken in this paper.

This paper is organized in six sections. Section 2 defines some notation that is used throughout the paper. Section 3 reviews some basic definitions in $\mathcal{H}_{2}$ IMF and gives an extension to the dynamic output feedback case. The formulation in this section provides a basis for the extension to the $\mu$ framework. Sections 4 and 5 review basic $\mu$ theories. Section 6 focuses on the solution of the robust IMF problem within the framework of $\mu$ theory. The paper finishes with an example illustrating the results developed.

\section{Notation}

The concept of linear fractional transformations (LFTs) are used throughout this paper. The LFT is defined on state-space representations of the form

$$
\begin{aligned}
P(s) & =\left[\begin{array}{l|l}
A & B \\
\hline C & D
\end{array}\right] \\
& =C(s I-A)^{-1} B+D
\end{aligned}
$$

A common use of (1) is for systems in which the coefficients, $A, B$, $C$, and $D$, have a partitioned structure. A commonly used structure is the following

$$
\begin{aligned}
G(s) & =\left[\begin{array}{c|cc}
A & B_{1} & B_{2} \\
\hline C_{1} & D_{11} & D_{12} \\
C_{2} & D_{21} & D_{22}
\end{array}\right] \\
& =\left[\begin{array}{ll}
G_{11}(s) & G_{12}(s) \\
G_{21}(s) & G_{22}(s)
\end{array}\right]
\end{aligned}
$$

The LFT is defined on (2), and can be thought of as closing loops with controllers or uncertainty perturbations. There are two general ways to close loops: either on the upper or lower block of (2). These transformations are defined as follows

$$
\begin{aligned}
& \mathcal{F}_{\ell}(G(s), K(s))=G_{11}+G_{12} K\left(I-G_{22} K\right)^{-1} G_{21} \\
& \mathcal{F}_{\mathrm{u}}(G(s), \Delta(s))=G_{22}+G_{21} \dot{\Delta}\left(I-G_{11} \Delta\right)^{-1} G_{12}
\end{aligned}
$$

where it is assumed that $G(s)$ is partitioned appropriately. The existence of the inverses is a necessary condition for (3) and (4) to be well defined; hence, we will always assume that the inverses exist whenever an LFT is considered. We will also assume that the functional dependence on $s$ is implicit when defining LFTs.

\section{$3 \quad \mathcal{H}_{2}$-optimal IMF with Output Feedback}

A new statement of earlier results in quadratic IMF is given in this section. The results of $[8]$ are extended to the dynamic output feedback case and reformulated in the $\mathcal{H}_{2}$ context.

Consider the linear system described by

$$
\begin{aligned}
& \dot{x}=A x+B u+a_{\text {per }} \\
& y=C x \\
& z=H x
\end{aligned}
$$

where $x \in \mathbb{R}^{n}$ is the state vector, $u \in \mathbb{R}^{m}$ is the input vector, $y \in$ $\mathbb{R}^{p}$ are the measured outputs, $z \in \mathbb{R}^{q}$ are the controlled outputs, $H \in \mathbb{R}^{q \times n}$ is full rank and selects the controlled modes from the state vector, and $d_{\text {per } f}$ is an exogenous signal of state initial conditions, namely

$$
d_{\text {perf }}=x(0) \delta(t)
$$

One would like to a find dynamic output feedback control law $u=K y$, for (5) such that the closed-loop dynamics of the controlled outputs $z$ are as close as possible to the desired system, given by

$$
\dot{\eta}=A_{d} \eta+H d_{\text {per } f}
$$

where $\eta \in \mathbb{R}^{q}$. Note that $A_{d}$ is usually selected to reflect time domain specifications in terms of settling time, damping ratio and state decoupling.

We now form the error derivative $\dot{e}=H \dot{x}-\dot{\eta}$. From (5) and (7) the error can be written

$$
\dot{e}=\left(H A x+H B u+H d_{\text {per } f}\right)-\left(A_{d} \eta+H d_{\text {perf }}\right)
$$

With the IMF paradigm, $\eta=H x$ [8], and substitution, (8) reduces to

$$
\dot{e}=\left(H A-A_{d} H\right) x+H B u
$$


The above problem can then be rewritten in quadratic terms in the form of a performance index as

$$
J_{2}=\int_{0}^{\infty}\left(\dot{e}^{T} R_{0} \dot{e}+u^{T} R_{1} u\right) d t
$$

where an input weight $R_{1}$ has been introduced for design flexibility. By substituting (5) into (10) this index becomes

$$
J_{2}=\int_{0}^{\infty}\left\{x^{T} R_{x} x+2 x^{T} R_{x u} u+u^{T} R_{u} u\right\} d t
$$

where

$$
\begin{aligned}
R_{x} & =\left(H A-A_{d} H\right)^{T} R_{0}\left(H A-A_{d} H\right) \\
R_{x u} & =\left(H A-A_{d} H\right)^{T} R_{0} H B \\
R_{u} & =(H B)^{T} R_{0}(H B)+R_{1}
\end{aligned}
$$

Thus, the $\mathcal{H}_{2}$-optimal IMF comes down to minimizing a standard type quadratic criterion but with a cross-weighted term $x^{T} R_{x u} u$. For convenience we define the following LFT system representation

$$
P_{2}(s)=\left[\begin{array}{c|cc}
A & B_{1} & B_{2} \\
\hline C_{1} & 0 & D_{12} \\
C_{2} & 0 & 0
\end{array}\right]
$$

where

$$
\begin{array}{ll}
B_{1}=I & C_{1}=\left[\begin{array}{c}
R_{0}^{1 / 2}\left(H A-A_{d} H\right) \\
0
\end{array}\right] \quad D_{12}=\left[\begin{array}{c}
R_{0}^{1 / 2} H B \\
R_{1}^{1 / 2}
\end{array}\right] \\
B_{2}=B & C_{2}=C
\end{array}
$$

Therefore, the $\mathcal{H}_{2}$-optimal IMF can be restated in the form of the minimization of

$$
K_{2}=\arg \inf _{K \text { stabilizing }}\left\|\mathcal{F}_{\ell}\left(P_{2}, K\right)\right\|_{2}
$$

Before continuing, it is useful to note that the $\mathcal{H}_{2}$-optimal IMF problem involves the solution of a singular problem because the measurements have been assumed to be noise free, (13). The $\mathcal{H}_{2}$-optimal controller achieving IMF is given by the following theorem which extends the results of [8].

Theorem $3.1\left(\mathcal{H}_{2}\right.$ IMF)

Define $X_{2} \geq 0$ and $Y_{2} \geq 0$ as solutions of the following Riccati equations

$$
\begin{aligned}
0= & A^{T} X_{2}+X_{2} A-X_{2} B_{2} B_{2}^{T} X_{2}+C_{1}^{T} C_{1} \\
0= & Y_{2} A^{T}+A Y_{2}+B_{1} B_{1}^{T} \\
& -\left(Y_{2} A^{T}+B_{1} B_{1}^{T}\right) C_{2}^{T}\left(C_{2} B_{1} B_{1}^{T} C_{2}^{T}\right)^{-1} C_{2}\left(A Y_{2}+B_{1} B_{1}^{T}\right)
\end{aligned}
$$

Define the state feedback matrix as

$$
F_{2}=-\left(D_{12}^{T} D_{12}\right)^{-1}\left(D_{12}^{T} C_{1}+B_{2}^{T} X_{2}\right)
$$

Then under the assumption that $C_{2} B_{1}$ is full rank, the reduced order $\mathcal{H}_{2}$-optimal controller achieving IMF is given by

$$
K_{2}=\left[\begin{array}{c|c}
\left(N A+N B F_{2}\right) M & \left(N A+N B F_{2}\right) \Xi \\
\hline F_{2} M & F_{2} \Xi
\end{array}\right]
$$

where

$$
\Xi=\left(Y_{2} A^{T}+B_{1} B_{1}^{T}\right) C_{2}^{T}\left(C_{2} B_{1} B_{1}^{T} C_{2}^{T}\right)^{-1}
$$

and $N$ and $M$ are matrices of appropriate dimensions solving

$$
\left[\begin{array}{c}
C_{2} \\
N
\end{array}\right][\Xi M]=I_{n}
$$

\section{Proof $3.1\left(\mathcal{H}_{2}\right.$ IMF $)$}

Note first that the full rank assumption of $C_{2} B_{1}$ is satisfied trivially for the IMF problem defined by $(13,14)$, with $B_{1}=I$ and $C_{2}=C$. Otherwise, derivatives of the measurements would be necessary, and the transfer function matrix of the observer becomes improper. It also ensures that the order of the observer is $n-p[2,7]$. Using a stochastic interpretation of the above $\mathcal{H}_{2}$ problem [3], the results of [1] yields the reduced-order optimal observer

$$
K_{\text {obs }}=\left[\begin{array}{c|cc}
N A M & N B & N A \Xi \\
\hline M & 0 & \Xi
\end{array}\right]
$$

where the output of the observer, $\hat{x} \in \mathbb{R}^{n}$ is the estimated state. Using the facts that the separation principle holds in this case [2] and that the LQ optimal static full-state feedback controller associated with (15) is given by (18), the controller, (19) follows immediately.

\section{Introduction to $\mu$ Analysis}

In this section we will briefly review the methods for analyzing the stability and performance properties of interconnected systems subject to norm-bounded structured uncertainty. Any linear interconnection of inputs, outputs, and uncertainty perturbations can be rearranged to fit the interconnection structure of Figure $1[4,5,6,9,10]$.

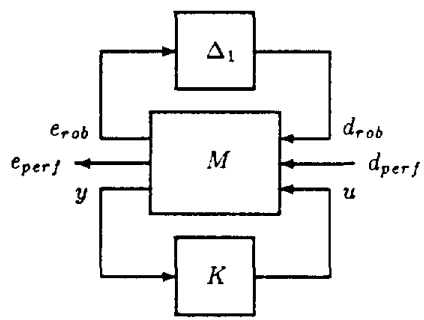

Figure 1: General Interconnection with Norm-Bounded Structured Uncertainty

$M$ describes a plant along with all the weighting functions on the inputs and outputs used to scale the norm-bounds to $1 . K$ is a cuiltroller mapping the measurements, $y$, to the plant control inputs, $u . \Delta_{1}$ is the norm-bounded structured uncertainty perturbation, and the mapping $d_{r o b} \rightarrow e_{\text {rob }}$ characterizes the uncertainty model being used. The mapping $d_{\text {per } f} \rightarrow e_{\text {perf }}$ characterizes the desired performance. The objective is to check if a controller, $K$, achieves robust performance, where robust performance is defined as achieving stability and performance over the entire set of norm-bounded perturbations. Hence, robust performance is achieved if $K$ stabilizes $\mathcal{F}_{\mathrm{u}}\left(M, \Delta_{1}\right), \forall \Delta_{1} \in \mathbf{B} \Delta_{1}$ and $\left\|\mathcal{F}_{\mathrm{u}}\left(\mathcal{F}_{l}(M, K), \Delta_{1}\right)\right\|_{\infty}<1, \forall \Delta_{1} \in$ $\mathbf{B} \Delta_{1}[5,6]$.

A matrix function $\mu$ will now be discussed which can be used to analyze the stability and performance properties of the interconnection structure in Figure 1.

Define $G=\mathcal{F}_{l}(M, K)$. Note that all of the matrices in Figure 1 are functions of a frequency parameter $\omega$. We will now close the loop from $e_{\text {perf }}$ to $d_{\text {perf }}$ with a complex full block and define the matrix function $\mu$ at a single frequency $\omega_{0}$.

Referring to Figure 2 we will evaluate $M, K$, and $\Delta$ at $\omega_{0}$. It will be assumed that $d_{\text {rob }}$ and $e_{\text {rob }}$ are of equal dimension $n_{1}$, and similarly that $d_{\text {perf }}$ and $e_{\text {perf }}$ are of equal dimension $n_{2}$. If this is not the case then augmentation of the rows or columns of $\mathcal{F}_{\ell}(M, K)$ by zeros wil' be used to force the previous conditions.

Let $F$ be the number of complex full blocks in the uncertainty perturbation $\Delta_{1}$. Then the set $\Delta_{1}$ is defined as

$$
\begin{aligned}
& \Delta_{1} \equiv\left\{\quad \Delta_{1}: \Delta_{1}=\text { block } \operatorname{diag}\left(\Delta_{k_{1}}, \ldots, \Delta_{k_{F}}\right),\right. \\
& \left.\Delta_{k_{i}} \in \mathbb{C}^{k, \times k,} i=1, \ldots, F\right\}
\end{aligned}
$$




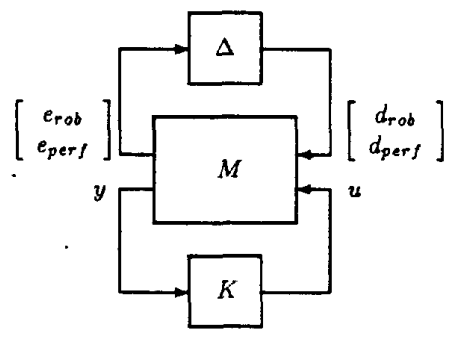

Figure 2: General Interconnection for $\mu$-analysis and -synthesis

where $\sum_{i=1}^{F} k_{i}=n_{1}$. Now the robust performance block structure can be defined by augmenting the uncertainty perturbation $\Delta_{1}$ by a complex full block

$$
\Delta \equiv\left\{\Delta: \Delta=\text { block } \operatorname{diag}\left(\Delta_{1}, \Delta_{2}\right), \Delta_{1} \in \Delta_{1}, \Delta_{2} \in \mathbb{C}^{n_{2} \times n_{2}}\right\}
$$

$\mu$ can now be defined on the robust performance block structure $\Delta$ as

Definition 4.1 ( $\mu$ for constant matrices [4])

$$
\mu_{\Delta}\left(G\left(\omega_{0}\right)\right)=\left\{\begin{array}{l}
\left(\min _{\Delta \in \Delta}\left\{\bar{\sigma}(\Delta): \operatorname{det}\left(I+G\left(\omega_{0}\right) \Delta\right)=0\right\}\right)^{-1} \\
0 \text { if no } \Delta \in \Delta \text { satisfies } \quad \therefore i\left(I+G\left(\omega_{0}\right) \Delta\right)=0
\end{array}\right.
$$

Now with the previous definitions in mind a precise definition of robust performance can be made.

Theorem 4.1 (Main Loop [9])

$$
\begin{aligned}
\mu_{\Delta}\left(G\left(\omega_{0}\right)\right)<1 \Longleftrightarrow & \mu_{\Delta_{1}}\left(G_{11}\left(\omega_{0}\right)\right)<1 \\
& \& \max _{\Delta_{1} \in \mathrm{B} \Delta_{1}} \mu_{\Delta_{2}}\left(\mathcal{F}_{\mathrm{u}}\left(G\left(\omega_{0}\right), \Delta_{\mathrm{I}}\right)\right)<1
\end{aligned}
$$

Where $\mathrm{B} \Delta_{1}=\left\{\Delta \in \Delta_{1}: \bar{\sigma}(\Delta)<1\right\}$ and $\mu_{\Delta_{3}}(\cdot) \equiv \bar{\sigma}(\cdot)$ because of the block structure of $\Delta_{2}$ [4].

If $\mu_{\Delta_{1}}\left(G_{11}\left(\omega_{0}\right)\right)<1$ and $G\left(\omega_{0}\right)$ is stable then there are no $\Delta_{1} \in \mathrm{B} \Delta_{1}$ which can destabilize $\mathcal{F}_{u}\left(G\left(\omega_{0}\right), \Delta_{1}\right)$ and if $\max _{\Delta_{1} \in B} \Delta_{1} \mu_{\Delta_{3}}\left(\mathcal{F}_{\mathrm{u}}\left(G\left(\omega_{0}\right), \Delta_{1}\right)\right)<1$ then performance is achieved at $\omega_{0}$ since $\frac{1}{\sigma}\left(\mathcal{F}_{\mathrm{u}}\left(G\left(\omega_{0}\right), \Delta_{1}\right)\right)<1 \quad \forall \Delta_{1} \in \mathrm{B} \Delta_{1}$. By a similar analysis it can be shown that if the right hand side of Theorem 4.1 is greater than or equal to one robust performance is not achieved. Hence. Theorem 4.1 demonstrates the equivalence of $\mu \Delta\left(G\left(\omega_{0}\right)\right)<1$ and robust performance at $\omega_{0}$

In the sequel it will be useful to establish an upper bound for $\mu$. With this in mind the following set is defined

$$
\begin{aligned}
& \mathcal{D} \equiv\left\{D \in \Delta: D=\text { block } \operatorname{diag}\left(d_{1} I_{k_{1}}, \ldots, d_{F} I_{k_{F}}, I_{n_{1}}\right),\right. \\
& \left.d_{i} \in \mathbb{R}, d_{i}>0, i=1, \ldots, F\right\}
\end{aligned}
$$

It is clear from (24) and (25) that elements of $\mathcal{D}$ and $\Delta$ commute. The upper bound for $\mu$ can now be written as:

Theorem 4.2 (Upper Bound [5, 9])

$$
\mu_{\Delta}\left(G\left(\omega_{0}\right)\right) \leq \inf _{D \in D} \bar{\sigma}\left(D G\left(\omega_{0}\right) D^{-1}\right)
$$

Henceforth it will be understood that all operations on matrices which are functions of $\omega$ will be shorthand for the sup over $\omega$. e.g. $\mu_{\Delta}(G) \equiv$ $\sup _{\Delta}(G(\omega))$.

\section{Introduction to $\mu$ Synthesis}

The following algorithm [5] will be used to find a controller satisfying $\mu_{\Delta}(G)<1$. It is assumed that the weighted open loop plant with the uncertainty and performance blocks (Figure 2) is given by $M$.

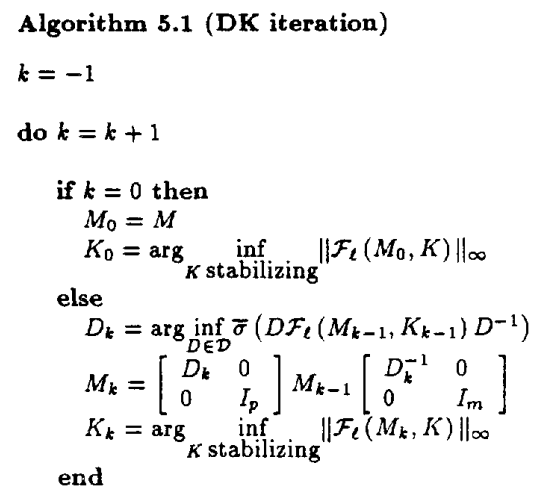

until $\left\|\mathcal{F}_{\ell}\left(M_{k}, K_{k}\right)\right\|_{\infty}-\left\|\mathcal{F}_{\ell}\left(M_{k-1}, K_{k-1}\right)\right\|_{\infty}<$ tol

It is noted that the $D$-scalings are computed as the argument of the infimum in the above algorithm, which yields a matrix of frequency varying data points. The multiplication of the $D$-scalings implied in the aigorithm is done before the frequency varying data is fit to a transier function. This prevents the states of the $D$-scalings from accumulating in each iteration of the algorithrn.

Since the infinity norm of the scaled system is norm nonincreasing we know the DK iteration will converge in a finite number of iterations. However, there is no a priori guarantee that the algorithm will converge to a global minimum. Suppose it converges on the $N^{\text {th }}$ iteration. If $\left\|\mathcal{F}_{\ell}\left(M_{N}, K_{N}\right)\right\|_{\infty}<1$, then, from Theorem 4.2 $\mu_{\Delta}\left(\mathcal{F}_{l}\left(M, K_{N}\right)\right)<1$ and, from Theorem $4.1, K_{N}$ achieves robust performance. If $\mu_{\Delta}\left(\mathcal{F}_{l}\left(M, K_{N}\right)\right) \geq l$ then the DK iteration must be repeated with the performance and uncertainty weights adjusted to restrict uncertainty or decrease performance.

\section{Robust IMF with $\mu$-synthesis}

The IMF Problem is concerned with achieving precise time domain requirements on a plant. Suppose we have the open loop system given by (5). As in the $\mathcal{H}_{2}$ formulation we would like to force certain modes of (5) to follow those of the reference model given by ( $\bar{i})$. In order to express IMF in the $\mu$ framework, $d_{\text {perf }}$ is now considered as an $\mathcal{L}_{2}(0,+\infty)$-integrable performance disturbance. We form the error derivative $\dot{e}=H \dot{\boldsymbol{x}}-\dot{\eta}$. From (5) and (7) the error equation can be written as (8). Employing the IMF paradigm, $\eta=H x$, the error equation reduces to (9); minimization of $\|\dot{e}\|_{2}$ will then implicitly assign the modes of $H x$

The IMF problem can be further augmented to include model uncertainty and is formulated as a $\mu$ problem with the following interconnection

$$
\begin{aligned}
M & =W_{\text {out }}\left[\begin{array}{c|ccc}
A & 0 & I & B \\
\hline H A-A_{d} H & 0 & 0 & I \\
0 & 0 & H B \\
C & -I & 0 & 0
\end{array}\right] W_{\text {in }} \\
W_{\text {out }} & =\left[\begin{array}{ccc}
W_{\text {out }}^{\text {oob }} & 0 & 0 \\
0 & W_{\text {out }}^{\text {perf }} & 0 \\
0 & 0 & I
\end{array}\right] \\
W_{\text {in }} & =\left[\begin{array}{ccc}
W_{\text {in }}^{\text {rob }} & 0 & 0 \\
0 & W_{\text {in }}^{\text {perf }} & 0 \\
0 & 0 & I
\end{array}\right]
\end{aligned}
$$


In the above interconnection structure the weights $W_{\text {out }}$ and $W_{\text {in }}$ are used to force the norm-bounds on the uncertainty and performance specifications to 1 . Comparison of (13) and (26) shows the similarity in the interconnection structures; they have the same peformance interconnection structure with the exception of the weighting on $u$ in the $\mathcal{H}_{2}$ formulation

The uncertainty model for this interconnection structure is given by the transfer function:

$$
d_{\text {rob }} \rightarrow e_{\text {rob }}=W_{o u t}^{r o b} K\left(I-C(s I-A)^{-1} B K\right)^{-1} W_{\text {in }}^{\text {rob }}
$$

The $\mathcal{H}_{\infty}$ norm of the transfer function in (27) for unstructured $\Delta_{1}$ characterizes additive uncertainty in the plant $M$, and the weights $W_{\text {out }}^{\text {rob }}$ and $W_{i n}^{\text {rob }}$ are generally chosen to reflect high-frequency uncertainty. This model can easily be modified to account for any structured uncertainty that might be present. The inclusion of structured uncertainty in the IMF structure will not change any of the results. The nominal performance is given by the transfer function $d_{\text {perf }} \rightarrow e_{\text {perf }}$ and the weights $W_{\text {out }}^{\text {per } f}$ and $W_{\text {in }}^{\text {per } f}$ are used to select the performance level in the region around the spectrum of $A_{d}$.

The perturbation structure is given by $F=1$, reflecting unstructured additive uncertainty; the block structure for the robust performance problem, given by (24), is composed of two complex full blocks, one for uncertainty and one for performance $\left(n_{2}=q\right)$.

The DK iteration defined in Algorithm 5.1 is used to obtain a controller achieving robust performance. As discussed in Section 5 the weights may have to be modified before the $D K$ iteration converges to a controller achieving robust performance.

\section{IMF Design Example}

\subsection{Model Development}

As an example, the IMF problem was considered for the longitudinal model of a helicopter. The model is 4-state, $\left[\begin{array}{llll}u & v_{z} & q & \theta\end{array}\right]^{T}$, and is given by the following

$$
\begin{aligned}
& A=\left[\begin{array}{cccc}
-0.0434 & 0.0116 & 0.0457243 & -0.1710297 \\
0.00487 & -0.976 & 0.9633508 & 0.0064921 \\
2.7446699 & -0.77928 & -2.03 & 0 \\
0 & 0 & 1 & 0
\end{array}\right] \\
& B=\left[\begin{array}{c}
0.1652705 \\
0.8324608 \\
-16.4 \\
0
\end{array}\right] \\
& C=\left[\begin{array}{llll}
0 & 0 & 1 & 0 \\
0 & 0 & 0 & 1
\end{array}\right]
\end{aligned}
$$

The desired modal characteristics of $g$ and $\theta$ are given by:

$$
A_{d}=\left[\begin{array}{cc}
-4.08 & -2.89 \\
1 & 0
\end{array}\right]
$$

$H$ is used to select the modal characteristics of $q$ and $\theta$, and is given by

$$
H=\left[\begin{array}{llll}
0 & 0 & 1 & 0 \\
0 & 0 & 0 & 1
\end{array}\right]
$$

The weights are given by

$$
\begin{aligned}
W_{\text {out }}^{\text {rob }} & =I_{1} \\
W_{\text {out }}^{\text {perf }} & =0.0152 \frac{(s+0.00001)(s+1000)}{(s+0.2)(s+8)} I_{2} \\
W_{\text {in }}^{\text {rob }} & =4.5 \frac{s+1}{s+20} I_{2} \\
Z_{\text {in }}^{\text {perf }} & =I_{4}
\end{aligned}
$$

These weights were chosen to reflect uncertainty in the high frequency dynamics and performance in the spectrum of $A_{d}$. The DK iteration converged to a controller achieving robust performance for these weights. $W_{i n}^{\text {rob }}$ and $W_{o u t}^{\text {perf }}$ are plotted on Figure 3.

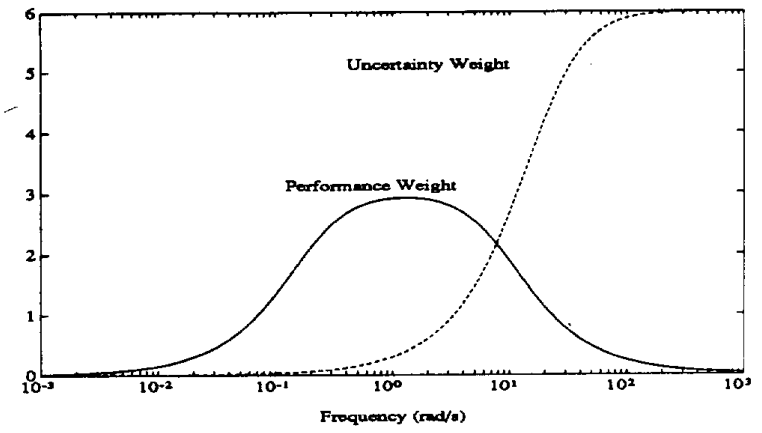

Figure 3: Robustness Weights

\subsection{Performance Comparison}

$\mathcal{H}_{2}, \mathcal{H}_{\infty}$ and $\mu$ synthesis methodologies were used to design controllers for the IMF problem. The $\mathcal{H}_{2}$ controller was designed using the interconnection matrix introduced in Section 3 . The $\mu$ controller was designed with the interconnection structure and weights introduced in Section 6 . The $\mathcal{H}_{\infty}$ controller was designed with the same interconnection structure as the $\mu$ controller without the uncertainty block. The $\mathcal{H}_{\infty}$ controller could be designed with this block included in the interconnection: this is equivalent to the controller resulting from the $k=0$ iteration in the DK synthesis algorithm. However, since $\mathcal{H}_{\infty}$ synthesis does not account for structured uncertainty this would equivalent to modelling all the uncerainty as a single complex full block, which is generally far too conservative.

The robustness properties of the different controllers are tabulated in Figure 4.

\begin{tabular}{|lr|c|c|c|}
\hline \multicolumn{2}{|c|}{ Measure } & \multicolumn{3}{c|}{ Controller } \\
\cline { 3 - 5 } & & $\mathcal{H}_{2}$ & $\mathcal{H}_{\infty}$ & $\mu$ \\
\hline Nominal Peformance & $\left\|G_{22}\right\|_{\infty}$ & 0.5402 & $8.5402 e-8$ & 0.7520 \\
Robust Stability & $\mu_{\boldsymbol{\Delta}_{1}}\left(G_{11}\right)$ & 1.3834 & 1.2967 & 0.7869 \\
Robust Peformance & $\boldsymbol{\mu}_{\boldsymbol{\Delta}}(G)$ & 1.3836 & 1.2968 & 0.8202 \\
\hline
\end{tabular}

Figure 4: Robustness Measures

Figures 5-7 graph the robustness properties of the different controllers. Each figure includes a graph of the nominal performance, robust stability, and robust performance measures which were quantified in Figure 4.

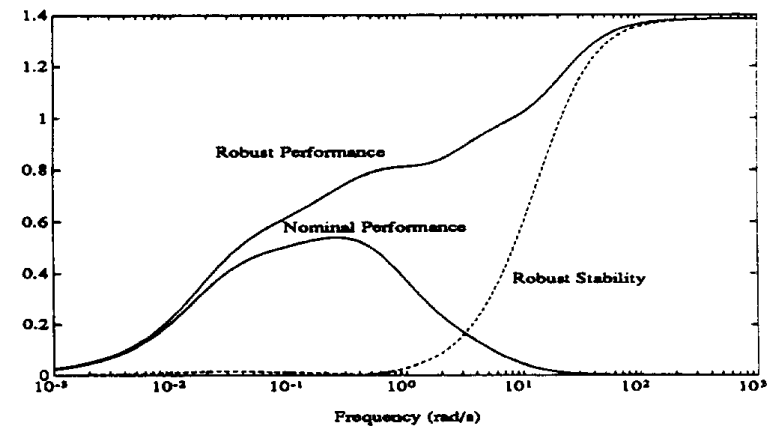

Figure 5: $\mathcal{H}_{2}$ Robustness

Referring to Figure 4, it is clear that all the controllers achieve nominal performance. Only the $\mu$ controller achieves robust stability and robust performance. The $\mathcal{H}_{\infty}$ controller clearly has the best 


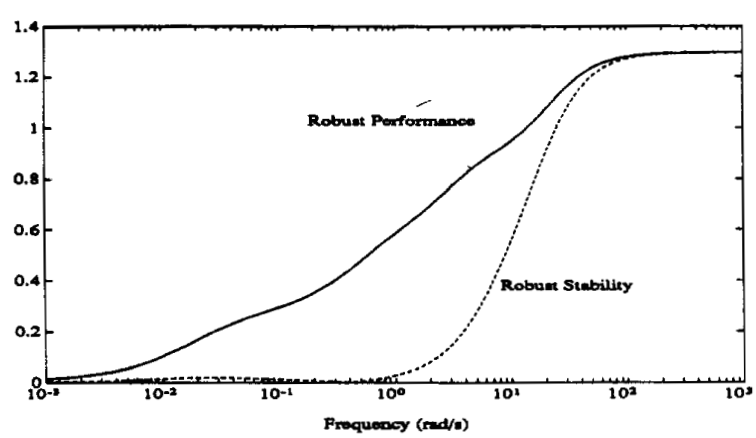

Figure 6: $\mathcal{H}_{\infty}$ Robustness

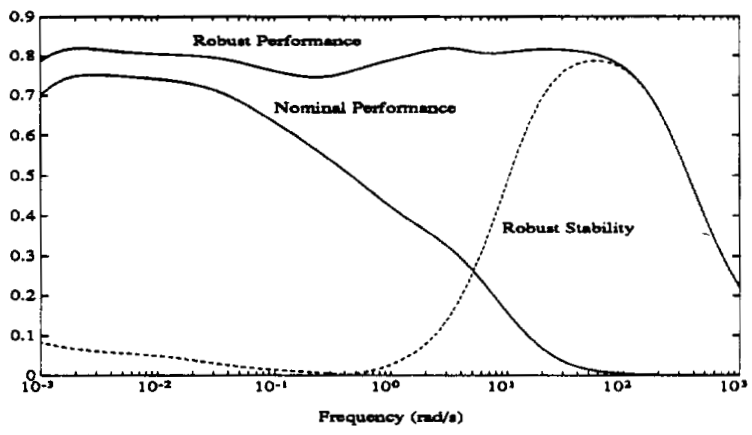

Figure 7: $\mu$ Robustness

nominal performance, and it will be shown to have the best nominal modal properties.

A time domain simulation was done for each of the closed-loop systems. Since the IMF formulation establishes modal properties of the closed-loop system and not steady-state properties, the unforced system response to changes in the initial conditions of the states selected by $H$ was simulated. For the $\mathcal{H}_{2}$ and $\mathcal{H}_{\infty}$ systems only the nominal response $\left(\Delta_{1}=0\right)$ to a step change in $\theta$ has been presented. For the $\mu$ system the nominal and perturbed response to step changes in both $q$ and $\theta$ have been presented. The perturbation used was the worst case performance perturbation normalized with $\left\|\Delta_{1}\right\|_{\infty}=1$ These simulations are graphed in Figures $8-13$. The graphs all include the desired response and the actual response for $q$ and $\theta$ as well as the error, defined as the difference between actual and desired.

Responses for perturbed $\mathcal{H}_{2}$ and $\mathcal{H}_{\infty}$ systems were not considered. From Figures 4-5 it can be seen that the overwhelming limitation with the $\mathcal{H}_{2}$ and $\mathcal{H}_{\infty}$ controllers is their inability to achieve robust stability. Hence, the perturbations of most significance are the destabilizing perturbations. Using the worst case destabilizing perturbation on both the $\mathcal{H}_{2}$ and $\mathcal{H}_{\infty}$ systems normalized so that $\left\|\Delta_{1}\right\|_{\infty}=1$ places unstable poles so far into the right half plane that simulation becomes intractable. Only the $\mu$ controller had sufficient robustness to stabilize the system and achieve the performance goal for all of the perturbations $\Delta_{1}$ in the block structure (23) satisfying $\left\|\Delta_{1}\right\|_{\infty}<1$.
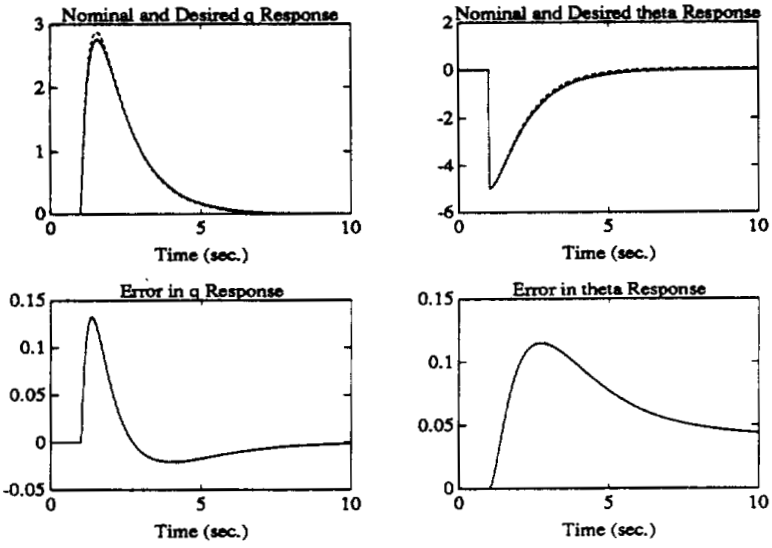

Figure 8: $\mathcal{H}_{2}$ Nominal Response to Step Change in $\theta$
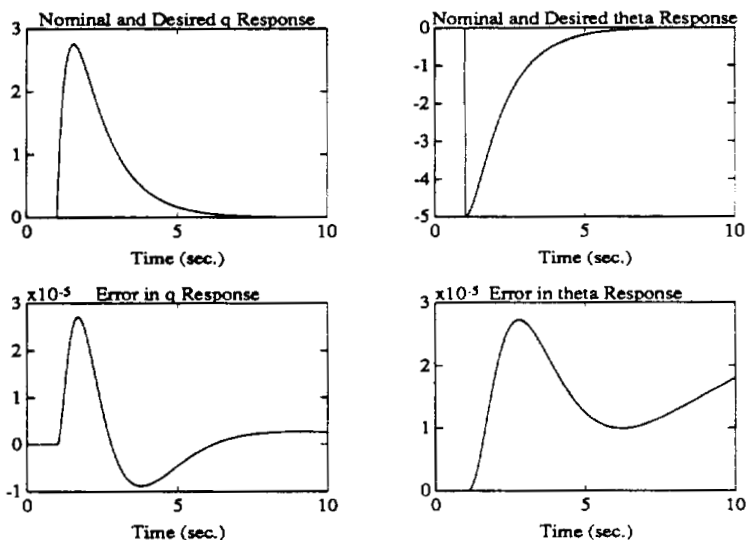

Figure 9: $\mathcal{H}_{\infty}$ Nominal Response to Step Change in $\theta$
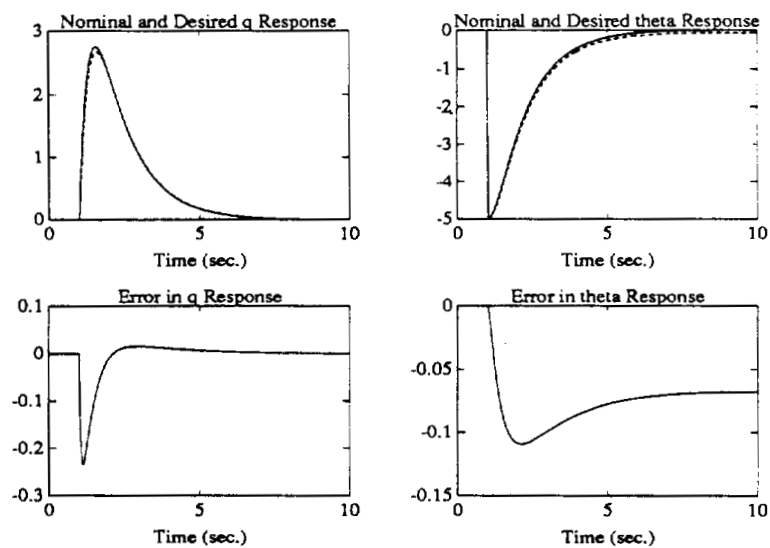

Figure 10: $\mu$ Nominal Response to Step Change in $\theta$ 

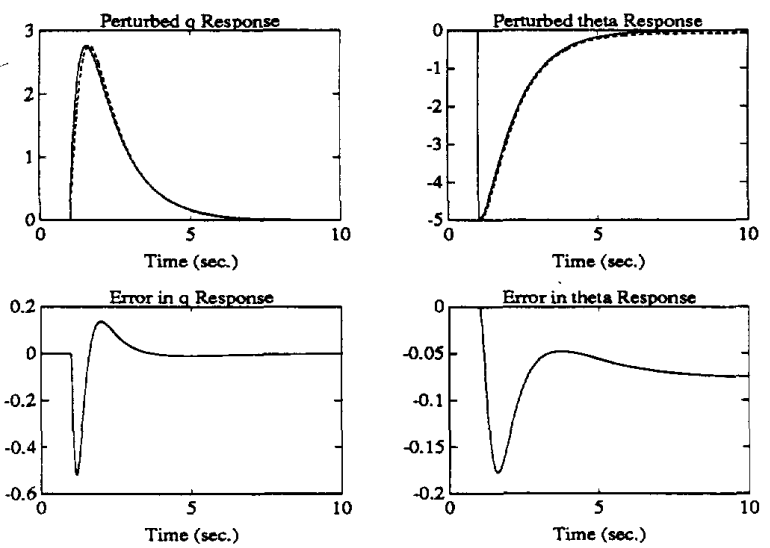

Figure 11: $\mu$ Perturbed Response System to Step Change in $\theta$
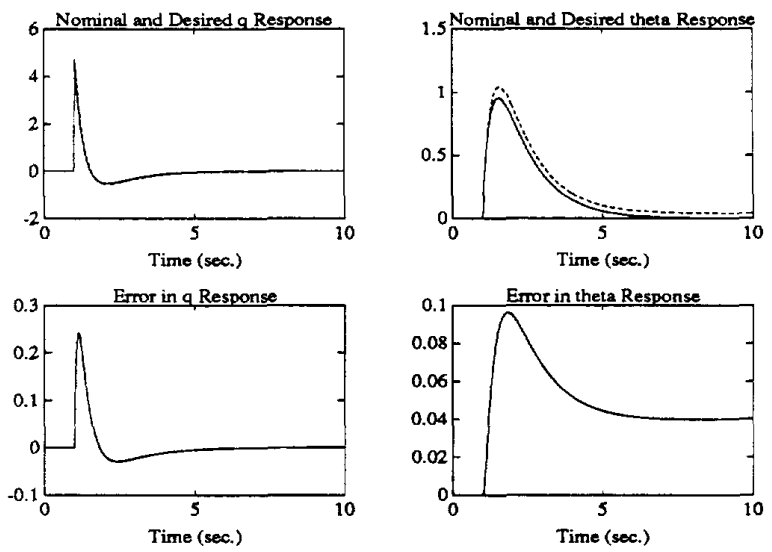

Figure 12: $\mu$ Nominal Response to Step Change in $q$
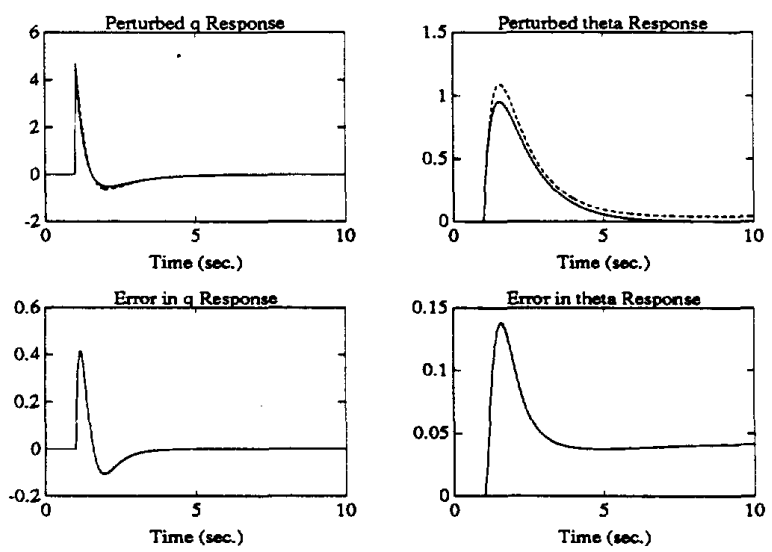

\section{Conclusions}

In this paper, the IMF problem has been considered and treated in the context of $\mathcal{H}_{2}, \mathcal{H}_{\infty}$, and $\mu$ control theories. New mixed performance/robustness problems, involving the IMF feedback structure subject to unstructured additive uncertainty have been developed. Extensions for structured uncertainties were discussed. The advantage of $\mu$-synthesis over $\mathcal{H}_{2}$ and $\mathcal{H}_{\infty}$ lies in an a priori robustness measure as a part of the synthesis procedure. The proposed $\mu$ control configuration has been shown to be very appealing in dealing with the issue of achieving robust state-space modal characteristics. Although the method is somewhat ad hoc its success on the helicopter problem in Section 7 encourages us to develop these ideas into a more systematic procedure for achieving robust time-domain performance.

\section{References}

[1] C. Champetier, "A duality principle for asymptotic regulator properties", Int. J. Contr., 38(3):547-556, 1983.

[2] D.S. Bernstein, Y. Halevi, and W.M. Haddad, "A riccati equation approach to the singular lqg problem", IEEE Auto. Contr. Conf. $(A C C)$, pp 2077-2078, 1989 .

[3] W.M. Haddad and D.S. Bernstein, "LQG control with a $\mathcal{H}_{\infty}$ performance bound: A riccati equation approach", IEEE Trans. Auio. Contr., AC-34(3):293-305, 1989.

[4] J.C. Doyle, "Analysis of feedback systems with structured uncertainties", IEE Proceedings, vol. 129, Part D, No ', pp. 242-250, Nov. 1982.

[5] J.C. Doyle, "Structured uncertainty in control system design", IEEE Conf. Decision Contr. (CDC), Dec. 1985.

[6] J.C. Doyle, K. Lenz, and A. Packard, "Design Examples using $\mu$ synthesis: Space Shuttle Lateral Axis FCS during reentry", IEEE CDC, Dec. 1986, pp. 2218-2223.

[7] Y. Halevi "The optimal reduced order estimator for systems with singular measurement noise", IEEE TAC, AC-34(7):777781 , July 1989.

[8] E. Kreindler and D. Rothschild, "Model-following in linearquadratic optimization", Journal of Guidance, Control and $D y$ namics", 14(7):835-842, July 1976.

[9] A.K. Packard, "What's new with $\mu$ : Structured Uncertainty in Multivariable Control", University of California, Berkeley, 1988

[10] J.E. Wall, J.C. Doyle, and G. Stein, "Performance _.ld robustness analysis for structured uncertainty", IEEE CDC, pp. 629-636, 1982.

Figure 13: $\mu$ Perturbed Response to Step Change in $q$ 